\title{
Assessment of Contextual Factors Shaping Delivery and Uptake of Isoniazid Preventive Therapy Among People Living with HIV in Dar Es Salaam, Tanzania.
}

Renatus Fabiano Nyarubamba ( $\nabla$ renny.fabby@gmail.com )

University of Zambia https://orcid.org/0000-0003-1620-1328

Adam Silumbwe

University of Zambia

Choolwe Jacobs

University of Zambia

Patricia Maritim

University of Zambia

Paschal Francis Mdoe

HLH: Haydom Lutheran Hospital

Joseph Mumba Zulu

University of Zambia

Research Article

Keywords: Isoniazid Preventive Therapy, Tuberculosis, HIV, implementation, Tanzania

Posted Date: February 18th, 2022

DOI: https://doi.org/10.21203/rs.3.rs-1198988/v1

License: (c) (1) This work is licensed under a Creative Commons Attribution 4.0 International License.

Read Full License 


\section{Abstract}

\section{Background}

Tuberculosis has remained a leading cause of deaths among People Living with HIV (PLHIV) globally. Isoniazid Preventive Therapy is the recommended strategy by the WHO to prevent TB disease and related deaths among PLHIV. However, delivery and uptake of IPT has remained suboptimal particularly in countries where HIV and TB are endemic such as Tanzania. The study sought to assess contextual factors that shape delivery of IPT in Dar Es Salaam region, Tanzania.

\section{Methodology}

A qualitative case study design was utilized whereby, in-depth interviews $(n=26)$ of clinicians and people living with HIV and Key Informat Interviews $(n=7)$ involving health administrators, were conducted. Thematic data analysis was done and reporting was guided by Consolidated Framework for Implementation Research (CFIR).

\section{Results}

Characteristics of intervention such as its adaptability to local contexts and relatively low cost of implementation facilitated its implementation. IPT was outsourced but this did not affect its implementation. On the contrary adverse effects of isoniazid that negatively affected implementation. Characteristics of individuals implementing the intervention including good knowledge and attitude towards IPT, commitment and efficacy of execution facilitated implementation. The process of IPT delivery that was characterized by engagement of stakeholders and implementors in planning facilitated implementation. Inner settings characteristics like connectedness among units, availability of resources and good implementation climate favoured implementation. Factors external to the implementing facilities such as networking of, and competitive pressure among facilities influenced delivery and uptake of IPT while HIV related stigma and some religious values stood as barriers.

\section{Conclusion}

Addressing policy related, procedural and individual barriers of IPT implementation at all levels of implementation is critical to effective implementation of this important intervention and ensuring all eligible people living with HIV are covered by IPT.

\section{Contribution To Literature}

- Poor delivery and uptake of IPT might partly explain the burden of TB related deaths among people living with HIV.

- Factors shaping delivery and uptake of IPT are applicable in other lower-middle-income countries thus, requiring attention of program managers policy makers, implementers and end users in order to improve success of this intervention. 
- Findings of this study can help to strengthen health systems through improving implementation of similar interventions in other similar settings.

\section{Introduction}

Tuberculosis (TB) and HIV co-infection remains a major public health problem globally and particularly in the developing world. Approximately 1.7 billion people are at risk of TB worldwide, 10 million fell ill in 2018 with $24 \%$ and $44 \%$ from Africa and South East Asia (1). HIV/AIDS has significantly fuelled TB incidences evidenced by 19 times higher likelihood of developing TB disease in PLHIV compared to those who are HIV negative. On the other hand, TB is the most common opportunistic infection and the major cause of deaths in PLHIV (1). In 2020, there were estimated 214,000 TB related deaths among PLHIV (2)

In Tanzania, an estimated 1.4 million people were living with HIV in 2018. Of these, 40,000 contracted TB and $40 \%$ died (1). The burden of TB among PLHIV in Tanzania was reported to be 16.7 cases per 1,000 person-years(3).

To reduce the burden of TB among PLHIV, the WHO recommended the use of Isoniazid Preventive Therapy (IPT) among PLHIV as a global public health strategy to reduce the incidences of TB in this subgroup of patients $(4,5)$. Isoniazid Preventive Therapy (IPT) is an evidence-based intervention that entails provision of Isoniazid (INH): one of the most effective bactericidal, anti-TB drugs, for a duration of six months after excluding active TB disease. In Tanzania, IPT was introduced in 2010 and scaled up nationwide in 2011.

Despite its effectiveness in preventing progression of latent TB infection into active TB disease, implementation of IPT has remained suboptimal. For example, in the sub-Saharan Africa region, South Africa had majority of newly diagnosed PLHIV put on IPT in 2019 at $69 \%$ and Eswatin had the lowest at about $1 \%(1,2)$. In Tanzania, only about $59.4 \%$ of patients eligible for IPT have been covered $(6)$. However, there is limited understanding of factors that shape delivery and uptake of IPT.

While elsewhere studies have looked at factors affecting implementation of IPT(7, 8, 9, 10). In Tanzania, studies have focused on determining the effectiveness of IPT, completion rate of IPT regimen, and knowledge on IPT $(11,12,13)$. There has been limited assessment of the contextual factors and they influence delivery and uptake of the IPT intervention. This study aimed to bridge this gap through assessing contextual factors that shape implementation of IPT intervention in the city of Dar Es Salaam Tanzania using Consolidated Framework for Implementation Research so as to inform policy guidelines towards effective implementation of IPT(14). CFIR has been widely used to study factors shaping implementation of various TB interventions(17).

\section{Methods}

\section{Study design}


A qualitative case study design was used because it was the most appropriate in describing IPT intervention, the contextual factors and how they shape implementation of this intervention (18).

\section{Study Setting}

This study was conducted in Dar Es Salaam, a major commercial city and major contributor of new TB cases in Tanzania with $20 \%$ of all TB cases in the country notified from this region in 2017 (19). National HIV impact survey (THIS) reported a regional HIV prevalence of $4.7 \%$ among adults 15 years and older (20). According to the 2012 National census, the city had a population of 4.3 million and thus, the most populous city in Tanzania, a literacy rate and employment rate of $97 \%$ and $61 \%$, respectively. In relations to TB/HIV services, in 2018 Dar Es Salaam had a total of 123 Care and treatment centers where IPT is offered.

\section{Study Participants}

Study participants comprised of 7 health administrators at different level of IPT implementation from the government and non-governmental organizations, 9 clinicians and 17 people living with HIV. Participants were purposively selected based on their experience in IPT services of at least one year and PLHIV were supposed to either be on or had completed IPT regimen. Except one who was approached by phone, the rest of participants were approached face to face and no one refused to participate in the study. All participants were at least 18 years old. Those who did not meet these criteria were excluded. Table 1 below, shows categories of participants.

Table 1

Categories of participants

\begin{tabular}{|lll|}
\hline S/No. & Participant category & Number of Interviews \\
\hline $\mathbf{1}$ & Health administrators & 7 \\
\hline $\mathbf{2}$ & Clinicians & 9 \\
\hline 3 & Patients/PLHIV & 17 \\
\hline TOTAL & & 33 \\
\hline
\end{tabular}

\section{Sampling Methods}

The study utilized purposive sampling methods to select participants and achieve a maximum-diversity sample. With help from the district health administration, Care and treatment centers were selected based on their performance in IPT implementation whereby those performing good and poorly were included in each of the five districts namely; Kigamboni, Temeke, llala, Ubungo and Kinondoni. Two care and treatment centers were recruited from each of the districts. 
While other participants were recruited based on their positions, patients were recruited at the clinics with assistance from TB/HIV focal person and clinic incharge who worked with the researcher to identify patients who were eligible for the study among those who attended clinics on the day of interview. However, the researcher had the final choice of which patient to be interviewed from among the ones recommended. Two patients were interviewed from each of the clinics.

Although we achieved saturation of data with about 25 interviews, we continued to a total of 33 interviews which however, did not reveal new information. The concept of "saturation", a point when incoming data produce little or no new information, is widely accepted as a standard by which sample size for qualitative inquiry are determined (21)

\section{Data Collection}

Having obtained permission to collect data from all the districts and selected clinics, we started collecting data through interviews and also patient's file were visited to confirm that a patient is on or has completed IPT regimen. Informed consent was sought before the interviews, which were conducted in a private room using a semi-structured questionnaire and lasted for utmost 60 minutes. The questionnaire was based on CFIR framework (https://cfirguide.org/guide/app/\#/guide_select). All the interviews were conducted face-to-face. However, given the COVID-19 pandemic, a distance of at least 2 meters between interviewer and the interviewee was observed. Moreover, interviewees were also encouraged to put on a mask as it was a case for the interviewer. Each interview was audio tapped and the interviewer took some notes whenever it was necessary. Data were collected between January and February, 2021.

\section{Data analysis}

This study employed thematic analysis approach, in which themes and patterns are identified, analyzed and presented from the data set (22). Recorded interviews were transcribed verbatim in Swahili language. Thereafter, English version of the transcripts were developed to enable review by a research team and for analysis. The transcripts were then read several times to develop codes from which themes were synthesized. Codes and themes were developed manually using the predetermined CFIR codebook to assess factors shaping delivery and uptake of IPT intervention (https://cfirguide.org/tools/tools-andtemplates/). Triangulation of information obtained in interviews and review of documents was done to improve trustworthiness of the study. Table 2 below shows a summary of major themes and subthemes that were developed. 
Table 2

Summary of themes and sub-themes

\begin{tabular}{|c|c|}
\hline Themes & Sub-themes \\
\hline \multirow[t]{5}{*}{ Characteristic of IPT intervention } & $\begin{array}{l}\text { - Sourced outside but endorsed } \\
\text { locally }\end{array}$ \\
\hline & - Effectiveness of IPT \\
\hline & - Adaptable to local contexts \\
\hline & $\begin{array}{l}\text { - Side effects, long queues, long } \\
\text { duration }\end{array}$ \\
\hline & - Not expensive \\
\hline \multirow{4}{*}{$\begin{array}{l}\text { Characteristics of individuals involved in delivery and uptake } \\
\text { of IPT }\end{array}$} & - Good knowledge and attitude \\
\hline & - Efficacy of delivering and using IPT \\
\hline & - Willing/stage to change \\
\hline & - HCWs commitment \\
\hline \multirow[t]{3}{*}{ Implementation processes of delivering IPT } & - Prior planning \\
\hline & - Engaging stakeholders \\
\hline & - Inclusive evaluation of progress \\
\hline \multirow[t]{4}{*}{ Internal settings } & $\begin{array}{l}\text { - Organizational arrangement of } \\
\text { units }\end{array}$ \\
\hline & $\begin{array}{l}\text { - Communication of goals and } \\
\text { feedback }\end{array}$ \\
\hline & - Availability of resources \\
\hline & - Supportive implementation climate \\
\hline \multirow[t]{4}{*}{ Outer settings } & $\begin{array}{l}\text { - Networking among facilities, } \\
\text { incentives }\end{array}$ \\
\hline & - HIV related stigma \\
\hline & $\begin{array}{l}\text {-Pressure among } \\
\text { organizations/facilities }\end{array}$ \\
\hline & - Cultural and religious values \\
\hline
\end{tabular}

\section{Results}

Many factors were identified to shape delivery and uptake of IPT in the region. The results of this study are presented below based on the five major domains of CFIR namely: characteristics of intervention, characteristics of individuals, process of implementing the intervention, inner settings and outer settings. 


\section{Characteristics of the IPT intervention}

Although it was adopted from the WHO, observed evidences of IPT in preventing active TB cases among PLHIV and adaptability of the intervention to local contexts led to it being supported by local actors such as healthcare providers, the media, government, civil society organizations and patients.

"We got guideline from WHO that eligible (HIV) clients should be started on IPT. Since we do follow and contextualize WHO directives, we started processes of implementing. Despite being sourced outside, I don't think this was of any problem because we as government and other stakeholders worked together on this like many other interventions"(Health Administrator)

IPT is provided for free of charge at all the clinics, like other TB and HIV related services, and this facilitated its implementation in the region. However, costs incurred monthly by patients to get to clinics, posed a barrier for some of them. Adverse side effects and complexities associated with the use of Isoniazid such as long duration, pill burden, long queues at the clinics and nausea and numbness discouraged some of the patients to undertake or complete IPT regimen.

"First of all, we take the drugs for long time-six months is longer. So, if possible, the duration perhaps should be reduced. Yes, you take once daily but for six months. It's so long. There are some people who can't take for all six months and thus stop taking the drugs" (Expert Client)

\section{Characteristics of Individuals involved in IPT delivery and uptake}

Majority of the participants demonstrated good knowledge about IPT in terms of what it is and that it is given to prevent progress from latent TB infection into active TB disease. Such understanding helped them to build trust and get committed towards the intervention and that opting to deliver or undertake IPT was the right thing do in order to reduce burden and deaths related to TB among PLHIV and protect the community.

"IPT is an intervention to protect people living with HIV from getting TB disease. IPT as IPT is provided to all eligible clients regardless of their age for a duration of six month" (Clinician)

However, despite being on IPT, few patients still could not tell exactly what it was and why were they given the drug. The confidence is one's ability to execute the intervention as guided by guidelines or clinicians, for patients, was critical towards implementation of IPT. When asked of how she understood IPT, one patient replied:

"I don't know what it means. We are just given drugs for prevention of Tuberculosis but I don't know what does that mean" (Patient)

\section{Organizational Processes Of Delivering Ipt Intervention}


Engagement of stakeholders including those at the lowest level of implementation and the patients in different processing of implementation and decision making such as planning, delivery of IPT and evaluation of implementation of the intervention in the region, not only promoted ownership but also facilitated implementation of IPT.

"Since I joined HIV services, I think one of the good things I have seen is that if there are changes, we clinicians are usually informed and our leaders will go to the meetings to plan how to adopt the changes in our works. I like that because it makes my work easy" (Clinician)

"There are monthly reports that indicate how many patients we have been able to cover and how many patients have completed the regimen within a particular month. So, every month we get a report on IPT completion" (Clinician)

\section{Characteristics within institutions delivering IPT intervention}

Close working connectedness among units within the facilities delivering IPT intervention such as laboratory unit, radiology unit and pharmacy and good communication among them helped to ensure uninterrupted and well-coordinated delivery of TB/HIV services including IPT.

“(...) every section does its job: laboratory, radiology unit and counselling. Every people play their roles to help patients. For me, I see a patient, listen to him and prescribe. But when I want blood tests, I send them outside to that room opposite to my office which is our Laboratory" (Clinician)

Implementation readiness characterised by availability of supplies such as drugs, financial and nonfinancial incentives and learning climate that enable sharing of experience and skills and delegation of tasks had positive influence for delivery of IPT to eligible patients.

"She (District HIV Coordinator), has a lot to do and meetings to attend. She sometimes cannot do all and she will ask some of us to do it. For example, today she is not here, she went to the other CTC for supervision so she asked me to prepare this report. (......) Of course, it feels good when you can also teach others since it makes you confident" (Clinician)

However, inadequate number of clinicians to some of the clinics left the present ones overwhelmed large number of patients to be served and thus prohibited effective delivery of IPT, among other TB/HIV related services.

"... when you go to facilities with enough staff, there is exit desk to ensure a client has received all and correct services and next appointment was provided. But now if I do all those processes, I forget offering IPT to a patient then I start looking for the file so as I can call the patient" (Clinician)

Factors external to the organizations delivering IPT. 
Facilities delivering IPT in the region have developed networking among themselves and this enables exchange of supplies such that deficit of supplies in one facility can not interrupt delivery of IPT. This networking has also caused competitive pressure among the facilities towards attaining targets which are set in collaboration between facilities, supporting organizations and authority in the region. All these combined largely influenced implementation of this intervention in the region.

"Cooperation between our facility and others exists and may include sharing of drugs. When they have no drugs, they will borrow from us because here drugs are easily available and one may tell you "Give me three boxes, I will bring it back when I receive my stock" (Clinician)

Although disclosing HIV status to relatives such as spouses and parents was generally accepted as important for getting treatment support, HIV stigma caused some of the patients not to do it which affected their uptake IPT. When asked whether it was important to disclose the HIV status, two patients said:

"Yes, it is very important (disclosing HIV status) because you may get sick or it may be your clinic visit date and someone should take you. You fail to ask someone to collect your drugs. It is very, very important but the question is how to get someone to disclose to. That's a challenge that gives me headache" (Patient)

To some of the patients, religious teachings were reported to influence their decision to uptake the intervention. Some of the HIV patients, despite being eligible for IPT, may refuse the intervention, believing that for them being religious, there was no chance to contract active TB disease. Describing an incidence that she once faced, one clinician narrated:

"(....) we used to tell her to bring back the empty packages for verification, now since she was a religious person, she could not lie. She used to keep the drugs at home and when she felt like we insisted much to see the used empty packages, she brought all the drugs: "here are your drugs, I am not taking it. I am (mentions her religion), I believe I won't suffer from TB disease" (Clinician)

\section{Discussion}

The study underscored several factors which shape delivery and uptake of IPT intervention. Active engagement of higher authorities appeared to affect IPT services at all levels of implementation in areas related advocacy, ensuring availability of supplies and providing mentorship, coaching and supervision. Evidence of the importance of leaders and managers in influencing IPT delivery and uptake was also revealed and reported in several other studies whereby lack of interaction with and support of manager overseeing HIV related care hindered effective implementation of IPT services(7, 23).

One of the key findings in our study was the importance of engaging the affected community to the success of IPT implementation. Champions of IPT use were engaged in TB/HIV services such as daily health talks, linking patients to services and even participating in counselling sessions. This finding 
reaffirmed what has been reported in other studies whereby using people from the affected community facilitated IPT implementation. A study in Cameroon reported how "expert clients" where found to play an important role in proper HIV related counselling (24), a stage which was also reported to be important towards uptake of IPT (25).

Integration of TB/HIV services with other routine services as well as providing all IPT related services under one roof reduces indirect costs and promotes delivery and uptake of the intervention. This surfaced in this study and added evidence on what has been reported from other studies. Providing IPT services and related services under one roof facilitated implementation of IPT services while poor integration of IPT services was a barrier to the implementation of the intervention $(7,8)$. Additional to integration, collaboration among healthcare providers in different units involving in IPT services implementation was of paramount importance (24) as it was also insisted in this study.

Knowledge, belief and attitude towards the intervention, like in many other studies, were also found to be key determinants of whether an intervention would be successful or not, in terms of its implementation. Good knowledge and attitude of patients and healthcare contributed to the delivery and uptake of IPT as reported in this study. However, it is important that these aspects go together for better outcomes because knowledge alone is not enough without the right attitude towards IPT among healthcare providers $(7,8)$. At the community level, when people share wrong belief regarding IPT, its implementation becomes difficult as it was reported in the study in South Africa whereby people believed that IPT introduced toxins in the body (9).

Characteristics of the intervention may act as barriers for its implementation. For example, the large number of Isoniazid pills to be taken, duration, associated side effects such as neuropathy and inconsistent availability of the drugs cause fear which result into both, patients and providers shying away from the intervention. Similar findings were reported in several studies conducted in other areas. In Kenya $(8,25)$ and (Omondi \& Wambiya, 2018) reported similar findings as it was the case in Ethiopia (23, 26) and (Mindachew et al., 2014).

Sharing experience and best practises and learning from other clinics implementing the same intervention has shown to play a significant role in improving implementation of the IPT intervention. Networking among clinics implementing IPT not only facilitated communication but also enabled exchange of drugs and other supplies in relation to IPT intervention and thus, ensured continuity of services. While this was found to be the case in our study, there are no many studies that have explored the role of networking although lack of such interoperability between clinics has been shown to hinder implementation of IPT services in yet another study conducted in Kenya (25).

Social support to PLHIV is very important to help them to successfully undertake treatment and interventions such as IPT as it was acknowledged by participants of this study. However, like in this study, HIV related stigma has been reported to hinder effective uptake of HIV related interventions such as IPT (24). Differently from these studies, HIV related stigma was reported to have no effect on IPT uptake (10). This could, however, be explained by differences in study settings whereby Jacobson et al conducted 
their study in subdistrict area which was characterized with poor socio-economic status, $80 \%$ rate of unemployment and only 15 primary health clinics indicating the likelihood of people in this area having less access to health services and education and thus unable to relate how HIV stigma could affect IPT services.

It's unfortunate that most of the people who are affected by HIV and thus, are at risk of developing TB disease, are in the sub-Saharan region where poverty is prevalent as well. Lack of financial resources was reported in this study as one of the barriers that hinders some patients to access and collect drugs as per clinic visit schedules. Similar findings were reported in studies conducted in South Africa whereby financial limitations and implications of not working for the sake of attending clinic visits affected uptake of IPT among people living with HIV $(9,10)$

\section{Conclusion}

Several factors shape delivery and uptake of IPT in the study area. These policy-related, procedural, structural and individual level factors need to be addressed through participation of various actors at all levels of implementation in order to ensure effective implementation of this intervention and coverage of all eligible PLHIV. This needs effort from the government, non-state actors, policy and decision makers as well as implementors and patients.

\section{Strengths And Limitations}

Given the ongoing COVID-19 pandemic, data collection through observation could not be carried out. However, many interviews were conducted to extract as much data as possible. The authors spent longer time in the study area to collect as much data as possible. Triangulation of data from interviews and document review improved trustworthiness of the study. Moreover, this study has added knowledge to and reaffirmed what was already regarding implementation of IPT which can help health policy makers, clinicians and patients to improve implementation of this intervention.

\section{Abbreviations}

AIDS- Acquired Immunodeficiency Syndrome

ART- Anti-Retroviral Therapy

CFIR-Consolidated Framework for Implementation Research

CTCs- Care and Treatment Centres (Clinics)

HIV- Immunodeficiency Virus

TB/HIV- Co-infection of HIV and Tuberculosis 
IDI- In Depth Interview

INH-Isoniazid

IPT: Isoniazid Preventive Therapy

LMICs-Lower- and Middle-Income Countries

MDH-Management and Development for Health

KII- Key Informant Interview

NIMR-National Institute for Medical Research

NGO- Non-Governmental Organizations

PLHIV-People Living with HIV

SDG-Sustainable Development Goal

TB-Tuberculosis

UNAIDS- United Joint Program for HIV/AIDS

UNZA-University of Zambia

WHO: World Health Organization

\section{Declarations}

\section{Ethical approval and consent to participate.}

Clearance to conduct this study was obtained from The University of Zambia Biomedical Research Ethical Committee (UNZABREC): REF. 1022-2020 and National Institute for Medical Research (NIMR) in Tanzania: NIMR/HQ/R.8a./VolIX/3541 Permission to conduct the study was also obtained from regional, district and facility authorities and informed consent was sought from participants before data collection.

\section{Permission to publish}

The permission to publish our findings was obtained from NIMR (Ref. NIMR/HQ/P.12 VOLXXXIII/117)

\section{Availability of data and materials}

The dataset during and/or analysed during the current study is available from the corresponding author on reasonable request. 


\section{Competing interest}

The authors declare to have no competing of interest.

\section{Funding}

Funds to conduct this study was obtained as part of the WHO/TDR Scholarship package which was granted to the first author to pursue a Master's degree of Public Health in Health Policy and Management at the University of Zambia, Lusaka, Zambia. Funders were not involved in any part of the study.

\section{Author's contribution}

The concept for this study was proposed by RFN and it was then refined in collaboration with JMZ and AS. Data collection was conducted by RFN. Analysis and writing manuscript were done by all authors. Review and editing of the report were done by JMZ, PM, AS and CJ. Project administration was done by RFN, CJ and PFM. Final report was reviewed and approved by all authors.

\section{Acknowledgement}

RFN is a recipient of a TDR scholarship under the Postgraduate Training Scheme in Implementation Research at the University of Zambia. We are grateful for the financial support for the training scheme provided by UNICEF, UNDP, World Bank and WHO Special Programme for Research and Training in Tropical Diseases (TDR).

I also would like to extend my thanks to my supervisors at the University of Zambia, Dr, Paschal Mdoe of Haydom Lutheran Hospital, Tanzania, NIMR and all participants of our study.

\section{References}

1. World Health Organization. (2019). Global Tuberculosis Report.

2. World Health Organization. (2021). Global Tubercuosis Report.

3. Majigo M, Somi G, Joachim A, Manyahi J, Nondi J, Sambu V, Rwebembera A, Makyao N. (2020). Prevalence and incidence rate of tuberculosis among HIV-infected patients enrolled in HIV care, treatment, and support program in mainland Tanzania. 1, 1-8.

4. Organization WH. \& UNAIDS. (1998). Policy statement on preventive therapy against tuberculosis in people living with HIV. Report of a meeting held in Geneva 18 - 20 February 1998. WHO Document, WHO/TB/98.(February), 1-26.

5. W.H.O. (2012). WHO policy on collaborative TB / HIV activities Guidelines for national programmes and other stakeholders. Creative Lynx, 330, 2-36. https://doi.org/23586124.

6. Ministry of health. Community Development, Gender, Elderly and Children (2020). National AIDS Control Programme, Prevention of Tuberculosis Among PLHIV. 
7. Wambiya E, Atela M, Eboreime E, Ibisomi L. Factors affecting the acceptability of isoniazid preventive therapy among healthcare providers in selected HIV clinics in Nairobi County, Kenya: a qualitative study. BMJ open. 2018;8(12):e024286. https://doi.org/10.1136/bmjopen-2018-024286.

8. Lester R, Hamilton R, Charalambous S, Dwadwa T, Chandler C, Churchyard GJ, Grant AD. (2010). Barriers to implementation of isoniazid preventive therapy in HIV clinics: A qualitative study. Aids, 24(SUPPL. 5), 45-48. https://doi.org/10.1097/01.aids.0000391021.18284.12.

9. Boffa J, et al. When prevention is dangerous: perceptions of isoniazid preventive therapy in KwaZuluNatal, South Africa. Public Health Action. 2019;9(1):24-31.

10. Jacobson KB, Niccolai L, Mtungwa N, Moll AP, Sheela V, Centre PC, Ferry T, Africa S, Ferry T, Africa S, Haven N. HHS Public Access. 2018;29(7):936-42. https://doi.org/10.1080/09540121.2017.1283390.

11. Sabasaba A, Mwambi H, Somi G, Ramadhani A, Mahande J, M. (2019). Effect of isoniazid preventive therapy on tuberculosis incidence and associated risk factors among HIV infected adults in Tanzania:A retrospective cohort study. BMC Infectious Diseases, 2019, 19-62. https://doi.org/10.1186/s12879-019-3696-x\%0A(2019).

12. Shayo GA, Moshiro C, Aboud S, Bakari M, Mugusi FM. Acceptability and adherence to Isoniazid preventive therapy in HIV-infected patients clinically screened for latent tuberculosis in Dar es Salaam, Tanzania. BMC Infect Dis. 2015;15(1):1-8. https://doi.org/10.1186/s12879-015-1085-7.

13. Munseri PJ, Talbot EA, Mtei L, Von Reyn CF. Completion of isoniazid preventive therapy among HIVinfected patients in Tanzania. International Journal of Tuberculosis Lung Disease. 2008;12(9):103741.

14. Damschroder LJ, Aron DC, Keith RE, Kirsh SR, Alexander JA, Lowery JC. (2009). Fostering implementation of health services research findings into practice: A consolidated framework for advancing implementation science. In Implementation Science (Vol. 4, Issue 1). https://doi.org/10.1186/1748-5908-4-50.

15. Probandari A, Widjanarko B, Mahendradhata Y, Sanjoto H, Cerisha A, Nungky S, Riono P, Simon S, Farid MN, Giriputra S, Putra AE, Burhan E, Wahyuni CU, Mustikawati D, Widianingrum C, Tiemersma EW. (2016). The path to impact of operational research on tuberculosis control policies and practices in Indonesia. 1(February).

16. Id CMY, Millones AK, Puma D, Jimenez J, Galea T, Id RC, Pages GS, Brooks MB, Lecca L, Nicholson T, Becerra MC, Keshavjee S. (2021). Closing delivery gaps in the treatment of tuberculosis infection: Lessons from implementation research in Peru. 1-16. https://doi.org/10.1371/journal.pone.0247411.

17. Ahmed AA, Grammatico M, Moll AP, Malinga S, Charalambous S, Ladines-lim JB, Jones J, Choi K, Shenoi SV, Ahmed AA, Grammatico M, Moll AP, Malinga S, Makhunga P, Charalambous S, Ladines$\lim$ JB, Jones J, Choi K. (2021). Factors associated with low tuberculosis preventive therapy prescription rates among health care workers in rural South Africa ABSTRACT. Global Health Action, 14(1). https://doi.org/10.1080/16549716.2021.1979281. 
18. Denzin NK, L. Y. (2011). The SAGE handbook of qualitative research. Thou sand-oaks; Sage.

19. National Tuberculosis and Leprosy Programme. (2016). Annual report for National TB and Leprosy Programme.

20. Tanzania HIV. Impact Survey (2019). Tanzania HIV Impact Survey: Population based HIV impact Assessment 2016-2017.

21. Guest G, Bunce A, Johnson L. How Many Interviews Are Enough?: An Experiment with Data Saturation and Variability. Field Methods. 2006;18(1):59-82.

22. Braun V, Clarke V. (2006). Using thematic analysis in psychology. 3, 77-101. https://doi.org/10.1177/1525822X05279903.

23. Teklay G, Teklu T, Legesse B, Tedla K, Klinkenberg E. Barriers in the implementation of isoniazid preventive therapy for people living with HIV in Northern Ethiopia: A mixed quantitative and qualitative study. BMC Public Health. 2016;16(1):1-9. https://doi.org/10.1186/s12889-016-3525-8.

24. Njozing BN, Edin KE, Sebastián MS, Hurtig A. (2011). Voices from the frontline : counsellors ' perspectives on TB / HIV collaborative activities in the Northwest Region, Cameroon.

25. Ngugi SK, Muiruri P, Odero T, Gachuno O. (2020). Factors affecting uptake and completion of isoniazid preventive therapy among HIV-infected children at a national referral hospital, Kenya : a mixed quantitative and qualitative study. 1-11.

26. Mindachew M, Deribew A, Memiah P, Biadgilign S. (2014). Perceived barriers to the implementation of isoniazid preventive therapy for people living with HIV in resource constrained settings: A qualitative study. In Pan African Medical Journal (Vol. 17). https://doi.org/10.11604/pamj.2014.17.26.2641. 